\title{
Higher Order Moment Processing of Laser Doppler Perfusion Signals
}

\author{
Mikael L. Arildsson, Karin Wårdell, and Gert E. Nilsson \\ Linköping University, Department of Biomedical Engineering, S-581 85 Linköping, Sweden \\ (Paper JBO-134 received Dec. 2, 1996; revised manuscript received June 16, 1997; accepted for publication June 19, 1997. )
}

\begin{abstract}
The laser Doppler technique is used to assess tissue perfusion. Traditionally an integrated, $\omega$-weighted (firstorder filter) power spectrum is used to estimate perfusion. In order to be able to obtain selective information about the flow in vessels with different blood cell velocities, higher order filters have been implemented, investigated, and evaluated. Theoretical considerations show that the output of the signal processor will depend on the flow speed, for a given concentration of blood cells, according to $S_{\text {out }} \propto v^{n}$ where $v$ is the average blood cell speed and $n$ is the spectral filter order. An implementation of filters using zero-, first-, second-, and third-order spectral moments was utilized to experimentally verify the theory by using a laser Doppler perfusion imager. Two different flow models were utilized: A Plexiglas model was used to demonstrate the various signatures of the power spectrum for different flow speeds and filter orders, whereas a Delrin model was used to study the relationship between the flow velocity and the output of the signal processor for the different filters. The results show good agreement with theory and also good reproducibility. Recordings made on the skin of the wrist area demonstrated that the flow in small veins can be visualized by the use of higher spectral orders. (c) 1997 Society of Photo-Optical Instrumentation Engineers. [S1083-3668(97)00204-9]
\end{abstract}

Keywords laser Doppler imaging; blood flow; higher order spectral filtering; power spectrum; compartment; microcirculation.

\section{INTRODUCTION}

Laser doppler perfusion imaging (LDPI) is a novel technology for visualization of tissue perfusion. ${ }^{1}$ By scanning a laser beam over the tissue surface and recording the rapid-intensity fluctuations in the backscattered light, information about the spatial distribution of blood flow is obtained. These intensity fluctuations are caused by the appearance of Doppler-shifted light produced by the interaction of light and moving blood cells in the tissue. When a scanning procedure is completed, the result is presented as a color-coded image on a monitor.

In the signal processor unit of the conventional LDPI system, the first moment of the power spectral density of the Doppler signal is continuously calculated and used as a measure of the perfusion. Theoretical considerations have shown that this moment of scales linearly with perfusion defined as the local speed times the concentration of blood cells under ideal circumstances. ${ }^{2}$ In real tissue, the situation is more complex, however, with different speed distributions of moving blood cells at different depths ${ }^{3}$ and microvascular compartments of the tissue. In addition, the multiple generation of Doppler-shifted light in tissue with a high concentration of blood cells results in nonlinearities and underestimation of the perfusion. ${ }^{4}$

Address all correspondence to Mikael Arildsson. Tel: 4613222 449; Fax: 4613101 902; E-mail: mikar@imt.liu.se
Using the first-order moment of the power spectral density as a base for the calculation of the perfusion algorithm gives an integrated measure of the average perfusion in the illuminated tissue volume, but renders no information about the quality of the perfusion. It is therefore not possible to gain information about the origin of the Doppler signal and whether this is generated by the blood cells moving at a slow speed in the nutritive capillary network, or by blood cells perfusing the shunting vessels and small arteries and veins at a higher speed. Information about the speed distribution is, however, intrinsically hidden in the signature of the Doppler spectral density. Proportionally more of the signal energy is allocated to the higher frequencies if the Doppler signal is generated mainly by high-velocity scatterers in the shunting vessels and small arteries, as opposed to a situation with dominantly lowvelocity scatterers, such as blood cells, perfusing a capillary network. By analyzing the shape of the power spectral density, information about the origin of the Doppler component as well as the quality of the perfusion can be obtained. One way of gaining such perfusion quality measures is to allocate spectral moments of a multitude of degrees and use these algorithms as the base for generation of perfusion images. ${ }^{5}$ In general a higher order moment will give a higher weight factor to blood cells moving at high velocity, while the zero-order moment

1083-3668/97/\$10.00 @ 1997 SPIE 
will result in an output signal reflecting the concentration of moving blood cells virtually independent of their velocity.

The aim of this study was to investigate how the use of higher order spectral moment filtering would affect the output of the signal processor, especially the possibilities of distinguishing among different blood cell speeds. Theoretical and practical considerations of using higher order filtering are also presented.

\section{THEORY}

This section discusses the theoretical aspects of using higher order spectral moments when filtering the backscattered laser light Doppler power spectrum. It is based on the theory presented by Bonner and Nossal ${ }^{2}$ and extends their theory from using only first-order spectral moment filtering to the $n^{\prime}$ th order.

The $n^{\prime}$ th order spectral moment can be described by the following equation:

$$
\left\langle\omega^{n}\right\rangle=\int_{-\infty}^{\infty}\left|\omega^{n}\right| S(\omega) \mathrm{d} \omega
$$

where $S(\omega)$ is the power spectrum of the detected photocurrent of the backscattered, Dopplerbroadened light. As shown by Bonner and Nossal, ${ }^{2}$ this can be rewritten as:

$$
\begin{aligned}
\left\langle\omega^{n}\right\rangle= & \frac{2 \beta}{\pi} \int_{0}^{\infty} \omega^{\prime \prime}\left(\int _ { 0 } ^ { \infty } \operatorname { c o s } ( \omega t ) \left\{\exp \left(2 \bar{m}\left[I_{1}(t)-1\right]\right)\right.\right. \\
& -\exp (2 \bar{m})\} \mathrm{d} t) \mathrm{d} \omega
\end{aligned}
$$

where $\beta$ is a constant in the range $0<\beta<1$, which describes the optical coherence at the detector, and $\bar{m}$ is the average number of collisions between a single photon and moving scatterers. The function $I_{1}(t)$ is the intermediate scattering function:

$$
I_{1}(t)=\frac{1}{1+\left(\left\langle v^{2}\right\rangle t^{2} / 12 \xi a^{2}\right)},
$$

where $a$ is the average radius of a spherical scatterer and $\xi$ is an empirically determined constant, depending on the shape of the moving blood cells. The integral described in Eq. (2) is easily evaluated using the substitutions $Z=\left(\sqrt{\left\langle v^{2}\right\rangle}\right) t / \sqrt{12 \xi} a$ and $\Omega$ $=\sqrt{12 \xi} a \omega / \sqrt{\left\langle v^{2}\right\rangle}$, which gives the following relationship:

$$
\begin{aligned}
\left\langle\omega^{n}\right\rangle= & \frac{2 \beta}{\pi}\left(\frac{\sqrt{\left\langle v^{2}\right\rangle}}{\sqrt{12 \xi} a}\right)^{n} \int_{0}^{\infty} \Omega^{n} \int_{0}^{\infty} \cos (\Omega Z) \\
& \times\left\{\exp \left(2 \bar{m}\left[I_{1}(Z)-1\right]\right)-\exp (-2 \bar{m})\right\} \mathrm{d} Z \mathrm{~d} \Omega \\
= & \left(\sqrt{\left\langle v^{2}\right\rangle}\right)^{n} f(\bar{m}) .
\end{aligned}
$$

Equation (4) shows that a signal processor using $n^{\prime}$ th order spectral filtering will generate an output proportional to the average speed of the scatterer raised to the power of $n$. The function $f(\bar{m})$ will be dependent only on the average number of collisions between a photon and a moving scatterer.

\section{IMPLEMENTATION}

For the implementation, a laser Doppler perfusion imager (PIM1.0, Lisca Development AB, Sweden) was modified to include spectral filtering of the zero, first, second, and third order (filters used: $\omega^{0}$, $\omega^{1}, \omega^{2}$, and $\left.\omega^{3}\right)$. In practice, the hardware signal processor was changed to implement the four different spectral order filters, and the system's software (LDI version 2.4, Lisca Development AB, Sweden) was changed to include noise correction and image generation for the three new signals. This made possible the presentation of four color images, representing the zero-, first-, second-, and third-order filtered power spectrum of the Doppler signal.

When measuring the Doppler power spectrum, thermal noise generated by the components of the signal processor and shot noise from the light detector are added to the spectrum. If the generated noise is regarded as independent of the Doppler power spectrum (it will, however, be dependent on the intensity of the backscattered light), the output of the signal processor, based on the $n^{\prime}$ th order spectral moment, can be described as:

$$
S_{\text {out }}=K \int_{\omega_{\min }}^{\omega_{\max }}\left[\omega^{n} P(\omega)+\omega^{n} R_{0}\right] \mathrm{d} \omega
$$

The second term is the shot noise generated by the light detector, which will be the main contributor of noise in the measured spectrum. The shot noise of the detector can be described by a constant $R_{0}$ independent of the angular frequency $\omega$ because the frequency distribution is considered to be Gaussian. The upper and lower limits of the integral have been taken to be $\omega_{\min }$ and $\omega_{\max }$, which correspond to the lower and upper bandwidth limits, respectively, of the signal processor. In the system used, these limits were set to $20 \mathrm{~Hz}$ and $13 \mathrm{kHz}$, respectively.

From Eq. (5) it can be concluded that for each increase in spectral order moment, the shot noise will be amplified by a factor $\left(\omega_{\max }-\omega_{\min }\right) /(n+1)$, when compared with the shot noise level of the previous, $n$ 'th spectral order moment. This implies a substantial increase in the noise level when increasing the order of the spectral filtering. Since the shot noise is only dependent on the intensity of the backscattered light, it can be compensated for by subtraction of a signal proportional to the photocurrent. 


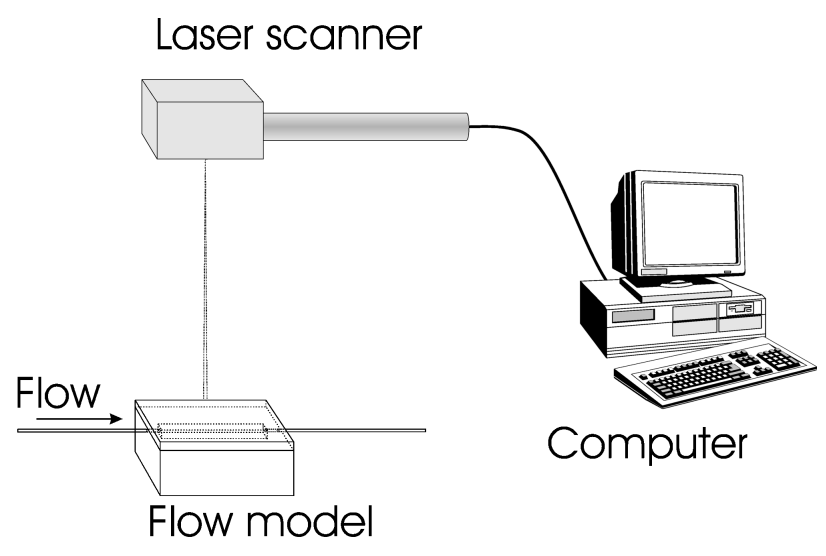

Fig. 1 The system setup during measurement. The flow model shown was used for in vitro measurement of blood flow and consists of a channel milled in a piece of Delrin. The channel is covered by a plate also made out of Delrin.

\section{EVALUATION}

Two different mechanical flow models were used to evaluate various aspects of the filters. The first model was used to investigate the power spectrum density of the laser Doppler signal after being fed through the different spectral order filters. The second model was used for in vitro measurements of blood flow in order to evaluate the performance of the filters when generating images with various flow velocities and blood concentrations. All measurements were made on human blood diluted in saline to a concentration of $0.1 \%$ or less. These low concentrations were used in order to minimize the influence of multiple scattering of the light by the moving blood cells.

During measurement, the filter output signals were simultaneously measured and displayed by a personal computer (DX2-66 MHz Ambra ${ }^{\mathrm{TM}}$, U.S.). The system setup during measurement is shown in Figure 1. The measurement data were then exported to MATLAB4.2 (The MathWorks Inc., U.S.) and EXCEL 7.0 (Microsoft Inc., U.S.) for analysis.

The first model was made of Plexiglas with a milled channel for guidance of the flow. A piece of semitransparent plastic was used to cover the channel and to scatter the incident laser light diffusely. An infusion pump (Sage Instrumentation's syringe pump Model 355, U.S.) was used to generate a blood flow (concentration $0.05 \%$ ) through the channel. The backscattered laser light power spectrum density, after being filtered through the different spectral order moment filters, was measured with a spectrum analyzer (HP35665A dynamic signal analyzer, Hewlett Packard, U.S.) using a flat-top window and averaging over 200 spectra. The bandwidth of the spectrum analyzer was chosen to be $12.8 \mathrm{kHz}$, which was found to be sufficient for the measurements in question. Noise corrections were made by turning off the blood flow through the fluid model and measuring the power spectrum density for the backscattered laser light. The recorded noise spectrum was then subtracted from the measured spectrum, which yielded the true Doppler spectrum. This method ensures that the noise spectrum will be measured under the same conditions as when there is flow through the flow model, i.e., that the intensity of the backscattered light will be the same and therefore the shot noise generated by the light detector will be the same in both cases. Three different flows (100, 200, and 400 $\mathrm{ml} / \mathrm{s}$ ) were used when measuring the different spectra. No images of the blood flow were recorded during these measurements.

A typical result from the measurements can be seen in Figure 2 in which the power spectral densities of the laser Doppler signal, after being processed by the four different filters, are shown for the different flows. Higher order filters demonstrate a movement of the center of gravity of the spectral densities toward higher frequencies. The higher frequencies also became more enhanced than lower frequencies. Furthermore, the bandwidth of the filtered signal increased radically with the higher order moment filters, especially for the third-order moment. This implies that a higher signal processor bandwidth should be used when using higher order filters.

Figure 3 shows the Doppler power spectral density of the backscattered light, both noise compensated and noise uncompensated, after being filtered through a second-order moment filter. As can be seen, the uncompensated power spectrum contains a substantial noise component that must be accounted for during a measurement.

The second model (shown in Figure 1) consists of a channel milled in Delrin ${ }^{\mathrm{TM}}$, a material with a similar scattering cross section, as can be seen in the cutaneous tissue matrix in which the blood vessels are embedded. The channel, $2 \mathrm{~mm}$ in width and 3 $\mathrm{mm}$ in height, was covered by a 1-mm-thick plate, also made of Delrin ${ }^{\mathrm{TM}}$. This ensures that the incident laser light is diffusely scattered before it interacts with the blood flow. Human blood diluted in saline was guided through the channel. The flow was generated by a hydrostatic pressure difference between the bowl holding the blood suspension and the flow model. Different flow velocities were generated by changing the hydrostatic pressure. The velocities ranged from 0 to $7 \mathrm{~cm}$ per second in steps of $1 \mathrm{~cm}$ per second. These settings ensured that the spectral power density was kept within the bandwidth of the signal processor. For each of the eight different flow velocities, 20 consecutive images for each of the different spectral filterings were recorded by the laser Doppler imager (a total of 640 images). The recorded images were exported to MATLAB. The 20 consecutive images recorded for each flow velocity were averaged into one single image, and the mean and standard deviation were calculated for all pixels $(n=64)$ located on the center line of the channel (i.e., each mean and standard 

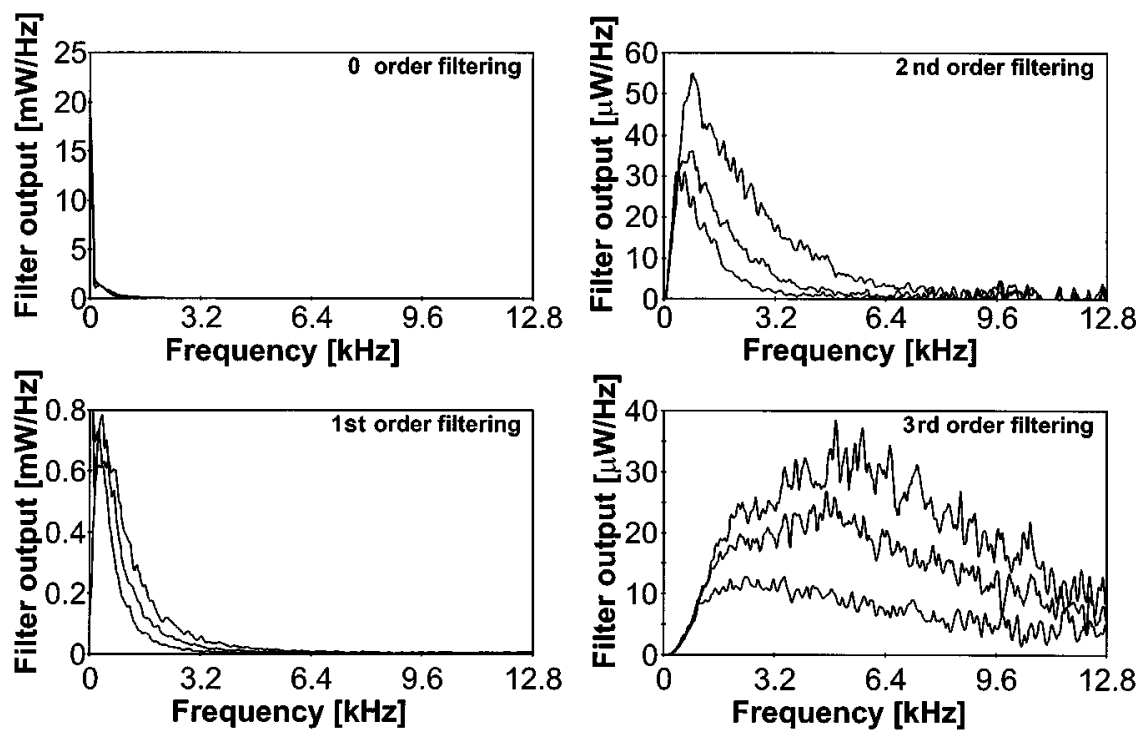

Fig. 2 Change in power spectrum of the output signal of the signal processor when using flows of 100, 200, and $400 \mathrm{ml} / \mathrm{s}$. Spectral filters used are zero, first, second, and third orders.

value was calculated from $64 \times 20=1280$ pixels). The data set was then exported to EXCEL for analysis. Figure 4 demonstrates the average measured output $(\mathrm{m}+\mathrm{SD})$ of the signal processor versus the flow velocity when using a blood concentration of $0.1 \%$. The measurements were found to show good reproducibility for different blood flow velocities.

To demonstrate the system's ability to create blood flow images with different weighting factors, a recording was made on the left wrist of a healthy male, using spectral filtering of the zero, first, second, and third order (see Figure 5-color plate). As the order of spectral filtering increases, a structure becomes visible (in the second- and third-order filtering), which could be identified as superficial veins on the subject's wrist. This behavior can be

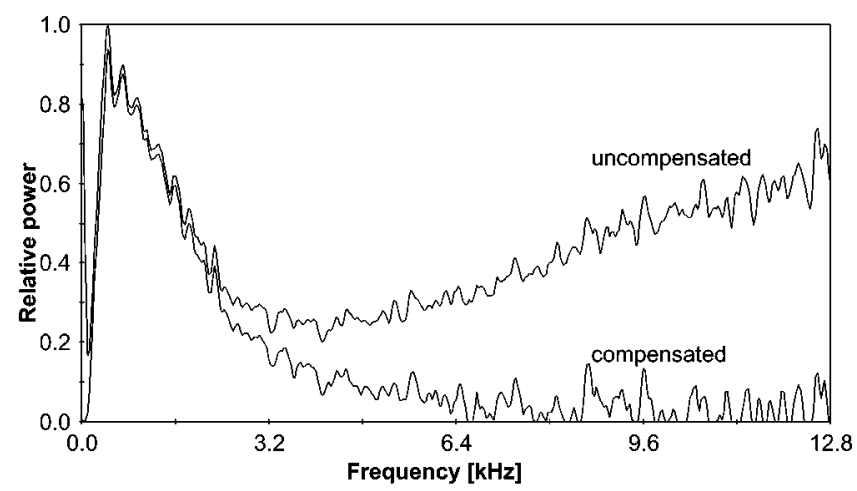

Fig. 3 The power spectrum of the filtered Doppler signal with and without noise compensation. The filter used is a second-order spectral filter. A noise component is found at $\mathrm{O} \mathrm{Hz}$ in the uncompensated power spectra due to an offset voltage in the signal processor filter. seen in all images recorded with the system; if a superficial vein could be seen by the eye, it was enhanced by the higher order filters.

\section{DISCUSSION}

Laser Doppler perfusion monitoring ${ }^{6,7}$ and imaging ${ }^{1}$ have up to now returned values that scale linearly with tissue blood perfusion defined as the product of the concentration and speed of moving blood cells within the scattering volume. Since the perfusion through different compartments of the tissue may vary widely and the photon density within the scattering volume is far from homoge-

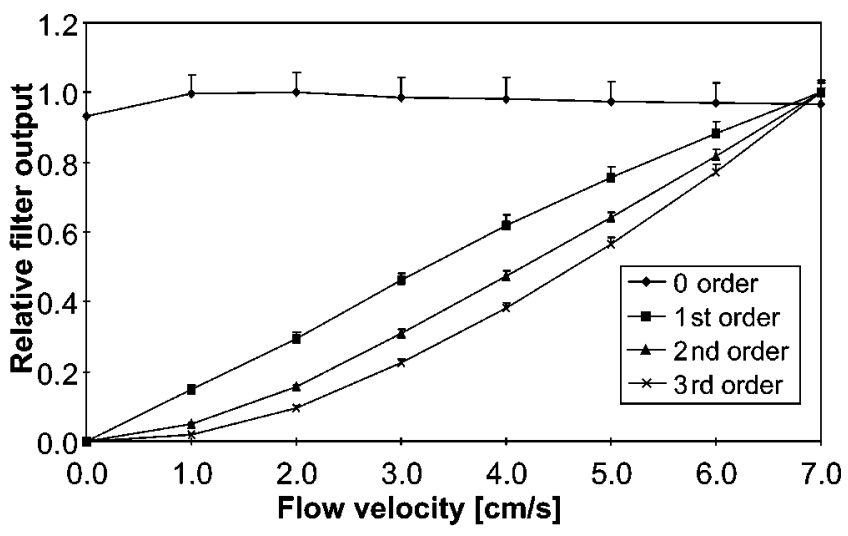

Fig. 4 The output of signal processors $(m \pm S D$ ) using spectral filtering of the zero, first, second, and third order for flow speeds in the range from 0 up to $7 \mathrm{~cm} / \mathrm{s}$ in steps of $1 \mathrm{~cm} / \mathrm{s}$. The measurement was made on a flow model made of Delrin using human blood diluted in saline to a concentration of $0.3 \%$. A laser Doppler perfusion imager was used to record the output of the signal processors. 


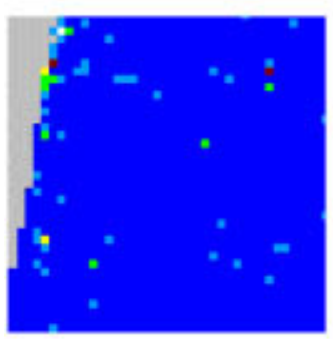

0 :th order filtering

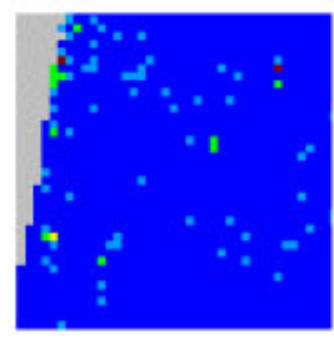

1 :st order filtering

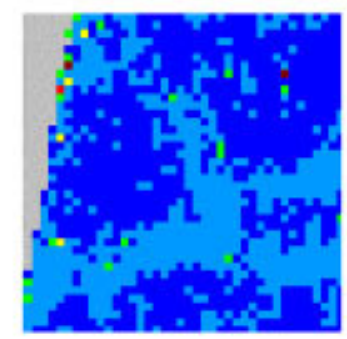

2:nd order filtering

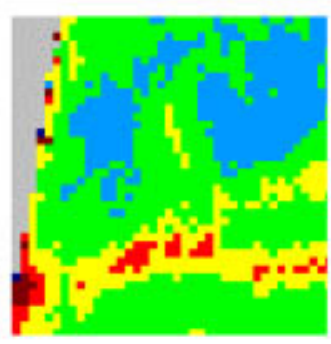

3:rd order filtering

\section{COLOR PLATE}

Fig. 5 Images recorded on the wrist of a healthy male subject using (a) zero-, (b) first-, (c) second-, and (d) third-order spectral filters. In the images recorded using higher order spectral filtering, a structure that can be identified as a superficial vein is visualized.

neous, the perfusion values that the laser Doppler devices deliver are to be interpreted and understood as average values representative for the tissue under study. With the standard signal processors in the laser Doppler equipment, no information can be obtained about the blood flow in the various compartments, e.g., the shunting vessels, the small arteries and veins, and the more superficial capillary network. In many experimental and clinical situations, however, qualitative information about the flow through different compartments is of paramount importance for the correct diagnosis of disease and follow up of treatment.

In this paper the focus has been on developing a method and a signal processor that utilizes the fact that the fast-moving blood cells and the local generation of multiple Doppler shifts in the small arteries and veins generally give rise to a shift in the bulk of the Doppler signal spectral density toward higher frequencies than what is generally the case for a capillary network perfused by slowly moving blood cells. By the use of filters that give higher weight factors to signals of higher frequencies, the perfusion through the different compartments can be assessed separately. Furthermore, by measuring the backscattered laser light in a large number of adjacent points and compiling the results to form an image by the use of a laser Doppler perfusion imager, it has proven to be possible to track nonoccluded blood vessels that can neither be observed by the naked eye nor detected by laser Doppler devices that calculate the first moment of the power spectral density alone.

In order to attain a high signal-to-noise ratio, the order of the filters is a critical design parameter that is dependent on the speed of the flow through the microvascular network under study. Lower order filters do not have the ability to extract the highspeed components from the bulk perfusion, while higher order filters suffer from a low signal-to-noise ratio because of the dominating power of the shot noise at higher frequencies. For the blood cell velocities generally encountered in a microvascular network, a filter based on the calculation of the third moment seems to be close to optimal.

The high noise level in the higher order signal processor is mainly due to the amplification by the higher order filters of the shot noise generated by the light detector. This shot noise can, in general, be regarded as white within the bandwidth of the signal processor, and is only dependent on the intensity of the backscattered light. This can be used to increase the signal-to-noise ratio by simply subtracting the shot-noise component from the output of the signal processor. However, owing to the imperfect integration of the filtered Doppler power spectrum by the signal processor, it will be virtually impossible to extract the Doppler signal completely from the noise; when using lower order filtering, the deviations of the measured stochastic noise from the expected value will be far less than for higher order filtering. The remedy for this problem is to increase the time constant of the integration, i.e., to integrate over a longer time period. For higher order filters, this approach would increase the time for a measurement substantially, which is unacceptable in the case of LDPI. The increased noise level of the measured spectra will also reduce the effective output swing of the signal processor. These factors will limit the maximum order of spectral filtering that can be employed to the third order (in the system used).

If a priori knowledge about the underlying microvascular network structure is at hand, a noninvasive system for automatic tracking and separation of occluded and nonoccluded vessels without the use of dyes and tracer elements can be anticipated to be particularly useful in the detection of occluded arteries in temporalis arteritis and in superficially positioned veins after reconstructive surgery. By utilizing lasers emitting radiation in the near-infrared region (780 to $960 \mathrm{~nm}$ ), the depth to which a vessel may be tracked can be altered and optimized. In combination with high-resolution la- 
ser Doppler perfusion imaging, ${ }^{8}$ the vessel tracker may furthermore be a useful tool in investigating the blood flow of the arteriovenous anastomosis of the diabetic foot.

\section{Acknowledgment}

This research was supported by the Swedish National Board for Technology Development, Grant P3735-1, compartment analysis.

\section{REFERENCES}

1. K. Wårdell, A. Jakobsson, and G. E. Nilsson, "Laser Doppler perfusion imaging by dynamic light scattering," IEEE Trans. Biomed. Eng. 40, 309-316 (1993).

2. R. Bonner and R. Nossal, "Model for laser Doppler measurements of blood flow in tissue," Appl. Opt. 20, 2097-2107 (1981).
3. A. Jakobsson and G. E. Nilsson, "Prediction of sampling depth and photon pathlength in laser Doppler flowmetry," Med. Biol. Eng. Comput. 31, 301-307 (1993).

4. H. Ahn, K. Johansson, O. Lundgren, and G. E. Nilsson, "In vivo evaluation of signal processors for laser Doppler tissue flowmeters," Med. Biol. Eng. Comput. 25, 207-211 (1987).

5. A. N. Obeid, "In vitro comparison of different signal processing algorithms used in laser Doppler flowmetry," Med. Biol. Eng. Comput. 31, 43-52 (1993).

6. G. E. Nilsson, T. Tenland, and P. A. Öberg, "A new instrument for continuous measurement of tissue blood flow by light beating spectroscopy," IEEE Trans. Biomed. Eng. 27, 12-19 (1980).

7. G. E. Nilsson, T. Tenland, and P. Å. Öberg, "Evaluation of a laser Doppler flowmeter for measurement of tissue blood flow," IEEE Trans. Biomed. Eng. 27, 597-604 (1980).

8. M. Lindén, A. Sirsjö, L. Lindbom, G. E. Nilsson, and A. Gidlöf, "Laser-Doppler perfusion imaging of microvascular blood flow in the rabbit tenussimus muscle," Am. J. Physiol. 269, 1496-1500 (1995) 